\title{
The * Congruence Class of the Solutions to a System of Matrix Equations
}

\author{
Yu-Ping Zhang and Chang-Zhou Dong \\ School of Mathematics and Science, Shijiazhuang University of Economics, Shijiazhuang 050031, China \\ Correspondence should be addressed to Chang-Zhou Dong; dongchangzhou@gmail.com
}

Received 14 May 2014; Revised 18 August 2014; Accepted 20 August 2014; Published 3 September 2014

Academic Editor: Qing-Wen Wang

Copyright (C) 2014 Y.-P. Zhang and C.-Z. Dong. This is an open access article distributed under the Creative Commons Attribution License, which permits unrestricted use, distribution, and reproduction in any medium, provided the original work is properly cited.

We present the ${ }^{*}$ congruence class of the least-square and the minimum norm least-square solutions to the system of complex matrix equation $A X=C, X B=D$ by generalized singular value decomposition and canonical correlation decomposition.

\section{Introduction}

Throughout we denote the complex $m \times n$ matrix space by $\mathbb{C}^{m \times n}$. The symbols $I, A^{*}$, and $\|A\|$ stand for the identity matrix with the appropriate size, the conjugate transpose, and the Frobenius norm of $A \in \mathbb{C}^{m \times n}$, respectively. Recall that matrices $X, Y \in \mathbb{C}^{n \times n}$ are in the same ${ }^{*}$ congruence class if there is a nonsingular $P \in \mathbb{C}^{n \times n}$ such that $X=P^{*} Y P[1]$.

Investigating the classical system of matrix equations

$$
A X=C, \quad X B=D
$$

has attracted many people's attention and many results have been obtained about system (1) with various constraints, such as Hermitian, positive definite, positive semidefinite, reflexive, and generalized reflexive solutions (see [2-10]). Studying the least-square solutions of the system of matrix equations (1) is also a very active research topic (see [11-16]). It is well known that Hermitian, positive definite and positive semidefinite matrices are the special case of ${ }^{*}$ congruence. Therefore investigating the * congruence class of a solution of the matrix equation (1) is very meaningful.

In 2005, Horn et al. [1] studied the possible ${ }^{*}$ congruence class of a square solution when linear matrix equation $A X=B$ is solvable. In 2009, Zheng et al. [17] describe * congruence class of least-square and minimum norm leastsquare solutions of the equation $A X=B$ when it is not solvable and discuss a ${ }^{*}$ congruence class of the solutions of the system (1) when it is solvable. To our knowledge, so far there has been little investigation of * congruence class of the least-square and minimum norm least-square solutions to (1) when it is not solvable.

Motivated by the work mentioned above, we investigate the ${ }^{*}$ congruence class of the least-square and the minimum norm least-square solutions to the system of complex matrix equation (1) by generalized singular value decomposition (GSVD) and canonical correlation decomposition (CCD).

\section{The * Congruence Class of the Solutions to (1)}

Lemma 1 (see [4]). Let $A \in \mathbb{C}^{m \times n}$ and $B \in \mathbb{C}^{n \times p}$. Then the GSVD of $A$ and $B^{*}$ can be expressed as

$$
A=U \Sigma_{A} P, \quad B^{*}=V \Sigma_{B} P,
$$

where $U \in \mathbb{C}^{m \times m}$ and $V \in \mathbb{C}^{p \times p}$ are unitary matrices, $P \in$ $\mathbb{C}^{n \times n}$ is nonsingular matrix,

$$
\begin{aligned}
& \Sigma_{A} \in \mathbb{C}^{m \times n}, \quad \Sigma_{B} \in \mathbb{C}^{p \times n}, \quad r=\operatorname{rank}\left(\begin{array}{c}
A \\
B^{*}
\end{array}\right), \\
& \Sigma_{A}=\left(\begin{array}{ccccc}
I_{A} & & & \cdot & \\
& S_{A} & & \cdot & 0 \\
& & O_{A} & \cdot &
\end{array}\right),
\end{aligned}
$$




$$
\Sigma_{B}=\left(\begin{array}{ccccc}
O_{B} & & \cdot & \\
& S_{B} & & \cdot & 0 \\
& & I_{B} & \cdot
\end{array}\right),
$$

$I_{A}$ and $I_{B}$ are identity matrices, $O_{A}$ and $O_{B}$ are zero matrices, and

$$
S_{A}=\operatorname{diag}\left(\alpha_{1}, \ldots, \alpha_{s}\right), \quad S_{B}=\operatorname{diag}\left(\beta_{1}, \ldots, \beta_{s}\right)
$$

with $1>\alpha_{1} \geq \cdots \geq \alpha_{s}>0,0<\beta_{1} \leq \cdots \leq \beta_{s}<1$, and $\alpha_{i}^{2}+\beta_{i}^{2}=1(i=1, \ldots, s)$.

For convenience, in the following theorem we denote

$$
\begin{aligned}
& P X P^{*}=\left(\begin{array}{cccc}
X_{11} & X_{12} & X_{13} & X_{14} \\
X_{21} & X_{22} & X_{23} & X_{24} \\
X_{31} & X_{32} & X_{33} & X_{34} \\
X_{41} & X_{42} & X_{43} & X_{44} \\
t & s & r-s-t & n-r
\end{array}\right. \\
& U^{*} C P^{*}=\left(\begin{array}{cccc}
C_{11} & C_{12} & C_{13} & C_{14} \\
C_{21} & C_{22} & C_{23} & C_{24} \\
C_{31} & C_{32} & C_{33} & C_{34}
\end{array}\right), \\
& P D V=\left(\begin{array}{lcc}
D_{11} & D_{12} & D_{13} \\
D_{21} & D_{22} & D_{23} \\
D_{31} & D_{32} & D_{33} \\
D_{41} & D_{42} & D_{43} \\
p-r+t & s & r-s-t
\end{array}\right.
\end{aligned}
$$

Theorem 2. Let $A, C \in \mathbb{C}^{m \times n}, B, D \in \mathbb{C}^{n \times p}$, and the GSVD of $A$ and $B^{*}$ be expressed as (2), and then one has the following.

(a) The system of matrix equation (1) has a solution in $\mathbb{C}^{n \times n}$ if and only if

$$
\begin{array}{cl}
C_{3 i}=0, \quad D_{i 1}=0, & (i=1,2,3,4), \\
C_{12}=D_{12} S_{B}^{-1}, & C_{13}=D_{13}, \\
S_{A}^{-1} C_{22}=D_{22} S_{B}^{-1}, & S_{A}^{-1} C_{23}=D_{23} .
\end{array}
$$

(b) In that case, the general solutions of (1) are

$$
X=P^{-1}\left(\begin{array}{cccc}
C_{11} & C_{12} & C_{13} & C_{14} \\
S_{A}^{-1} C_{21} & S_{A}^{-1} C_{22} & D_{23} & S_{A}^{-1} C_{24} \\
X_{31} & D_{32} S_{B}^{-1} & D_{33} & X_{34} \\
X_{41} & D_{42} S_{B}^{-1} & D_{43} & X_{44}
\end{array}\right)\left(P^{-1}\right)^{*},
$$

where $X_{31}, X_{41}, X_{34}$, and $X_{44}$ are arbitrary.

(c) For arbitrary $X_{31}, X_{41}, X_{34}$, and $X_{44}$, there exists a solution in $\mathbb{C}^{n \times n}$ of (1) which is * congruent to

$$
Y=\left(\begin{array}{cccc}
C_{11} & C_{12} & C_{13} & C_{14} \\
S_{A}^{-1} C_{21} & S_{A}^{-1} C_{22} & D_{23} & S_{A}^{-1} C_{24} \\
X_{31} & D_{32} S_{B}^{-1} & D_{33} & X_{34} \\
X_{41} & D_{42} S_{B}^{-1} & D_{43} & X_{44}
\end{array}\right)
$$

(d) There exists a minimum norm solution in $\mathbb{C}^{n \times n}$ of (1) which is ${ }^{*}$ congruent to

$$
Y=\left(\begin{array}{cccc}
C_{11} & C_{12} & C_{13} & C_{14} \\
S_{A}^{-1} C_{21} & S_{A}^{-1} C_{22} & D_{23} & S_{A}^{-1} C_{24} \\
0 & D_{32} S_{B}^{-1} & D_{33} & 0 \\
0 & D_{42} S_{B}^{-1} & D_{43} & 0
\end{array}\right)
$$

Proof. Using the GSVD of $A$ and $B^{*}$ given by (2), we get

$$
\begin{aligned}
& A X=C \Longleftrightarrow U \Sigma_{A} P X=C \Longleftrightarrow \Sigma_{A} P X P^{*}=U^{*} C P^{*}, \\
& X B=D \Longleftrightarrow X P^{*} \Sigma_{B}^{*} V^{*}=D \Longleftrightarrow P X P^{*} \Sigma_{B}^{*}=P D V .
\end{aligned}
$$

By (2) and (5), $\Sigma_{A} P X P^{*}$ and $P X P^{*} \Sigma_{B}^{*}$ have the following matrix decomposition:

$$
\begin{gathered}
\Sigma_{A} P X P^{*}=\left(\begin{array}{cccc}
X_{11} & X_{12} & X_{13} & X_{14} \\
S_{A} X_{21} & S_{A} X_{22} & S_{A} X_{23} & S_{A} X_{24} \\
0 & 0 & 0 & 0
\end{array}\right), \\
P X P^{*} \Sigma_{B}^{*}=\left(\begin{array}{ccc}
0 & X_{12} S_{B} & X_{13} \\
0 & X_{22} S_{B} & X_{23} \\
0 & X_{32} S_{B} & X_{33} \\
0 & X_{42} S_{B} & X_{43}
\end{array}\right),
\end{gathered}
$$

and we have that system (1) is equivalent to

$$
\begin{gathered}
\left(\begin{array}{cccc}
X_{11} & X_{12} & X_{13} & X_{14} \\
S_{A} X_{21} & S_{A} X_{22} & S_{A} X_{23} & S_{A} X_{24} \\
0 & 0 & 0 & 0
\end{array}\right)=\left(\begin{array}{llll}
C_{11} & C_{12} & C_{13} & C_{14} \\
C_{21} & C_{22} & C_{23} & C_{24} \\
C_{31} & C_{32} & C_{33} & C_{34}
\end{array}\right) \\
\left(\begin{array}{cccc}
0 & X_{12} S_{B} & X_{13} \\
0 & X_{22} S_{B} & X_{23} \\
0 & X_{32} S_{B} & X_{33} \\
0 & X_{42} S_{B} & X_{43}
\end{array}\right)=\left(\begin{array}{lll}
D_{11} & D_{12} & D_{13} \\
D_{21} & D_{22} & D_{23} \\
D_{31} & D_{32} & D_{33} \\
D_{41} & D_{42} & D_{43}
\end{array}\right) ;
\end{gathered}
$$

obviously, the system of matrix equation (1) has a solution in $\mathbb{C}^{n \times n}$ if and only if

$$
\begin{array}{r}
C_{3 i}=0, \quad D_{i 1}=0, \quad S_{A} X_{2 i}=C_{2 i}, \\
X_{i 2} S_{B}=D_{i 2}, \quad X_{1 i}=C_{1 i}, \quad X_{i 3}=D_{i 3}, \\
(i=1,2,3,4) .
\end{array}
$$

Therefore, (1) has a solution in $\mathbb{C}^{n \times n}$ if and only if (7) holds, and a general form of the solutions can be expressed as (8); for arbitrary $X_{31}, X_{41}, X_{34}$, and $X_{44}$, there exists a solution in $\mathbb{C}^{n \times n}$ of (1) which is * congruent to (9), and the part (d) follows from the definition of Frobenius norm.

Remark 3. In 2009, Zheng et al. [17] discuss a * congruence class of the solutions of the system (1) when it is solvable. Our result in Theorem 2 is different with the result mentioned above. 


\section{The * Congruence Class of the Least-Square Solutions to (1)}

Lemma 4 (see [18]). Let the CCD of matrix pair $[A, C]$ with $A \in \mathbb{C}^{m \times n}, C \in \mathbb{C}^{m \times k}, \operatorname{rank} A=g$, and $\operatorname{rank} C=h$ be given as

$$
A=U\left(\Sigma_{A}, 0\right) E_{A}^{-1}, \quad C=U\left(\Sigma_{C}, 0\right) E_{C}^{-1},
$$

where $U$ is a unitary matrix and

$$
\Sigma_{A}=\left(\begin{array}{ccc}
I_{i} & & \\
& \Lambda_{j} & \\
\overline{0} & - & \underline{0} \\
& \Delta_{j} & \\
& & I_{t}
\end{array}\right), \quad \Sigma_{C}=\left(\begin{array}{c}
I_{h} \\
\overline{0}
\end{array}\right)
$$

are nonsingular matrices with the same row partitioning, and $g=i+j+t$,

$$
\begin{aligned}
& \Lambda_{j}=\operatorname{diag}\left(\lambda_{i+1}, \ldots, \lambda_{i+j}\right), \quad 1>\lambda_{i+1} \geq \cdots \geq \lambda_{i+j}>0, \\
& \Delta_{j}=\operatorname{diag}\left(\Delta_{i+1}, \ldots, \Delta_{i+j}\right), \quad 0>\Delta_{i+1} \geq \cdots \geq \Delta_{i+j}>1, \\
& \Lambda_{j}^{2}+\Delta_{j}^{2}=I_{j} \text {, } \\
& \left.U=\begin{array}{cccccc}
u_{1} & u_{2} & u_{3} & u_{4} & u_{5} & u_{6}
\end{array}\right)
\end{aligned}
$$

Lemma 5 (see [18]). Given $E, F \in \mathbb{C}^{m \times n}$, then there exists a unique matrix $\bar{S} \in \mathbb{C}^{m \times n}$ such that

$$
\|S-E\|^{2}+\|S-F\|^{2}=\min ,
$$

and $\bar{S}$ can be expressed as

$$
\bar{S}=E+F .
$$

Lemma 6 (see [10]). Given $E, F \in \mathbb{C}^{m \times n}, \Omega_{1}=$ $\operatorname{diag}\left(a_{1}, \ldots, a_{m}\right), \Omega_{2}=\operatorname{diag}\left(b_{1}, \ldots, b_{n}\right), a_{i}>0(i=1, \ldots, m)$, and $b_{j}>0(j=1, \ldots, n)$, then there exists a unique matrix $\bar{S} \in \mathbb{C}^{m \times n}$ such that

$$
\left\|\Omega_{1} S-E\right\|^{2}+\left\|S \Omega_{2}-F\right\|^{2}=\min ,
$$

and $\bar{S}$ can be expressed as

$$
\bar{S}=\Phi *\left(\Omega_{1} E+F \Omega_{2}\right),
$$

where

$$
\Phi=\left(\frac{1}{a_{i}^{2}+b_{j}^{2}}\right) \in \mathbb{C}^{m \times n} .
$$

Using Lemmas 5 and 6, we can easily obtain the following.

Lemma 7. Given $E, F, G \in \mathbb{C}^{m \times n}, \Omega_{1}=\operatorname{diag}\left(a_{1}, \ldots, a_{m}\right)$, $\Omega_{2}=\operatorname{diag}\left(b_{1}, \ldots, b_{m}\right), I_{n}=\operatorname{diag}\left(i_{1}, \ldots, i_{n}\right), a_{i}>0(i=$ $1, \ldots, m), b_{j}>0(j=1, \ldots, m)$, and $i_{k}=1(k=1, \ldots, n)$, then there exist unique matrices $S$ and $W$ such that

$$
\left\|\Omega_{1} S+\Omega_{2} W-E\right\|^{2}+\|S-F\|^{2}+\|W-G\|^{2}=\min ,
$$

and $S$ and $W$ can be expressed as

$$
S=F, \quad W=\Phi *\left(\Omega_{2}\left(\Omega_{1} F-E\right)+G\right),
$$

where

$$
\Phi=\left(\frac{1}{b_{j}^{2}+i_{k}^{2}}\right) \in \mathbb{C}^{m \times n} .
$$

Lemma 8. Given $E, F \in \mathbb{C}^{m \times n}, \Omega_{1}=\operatorname{diag}\left(a_{1}, \ldots, a_{m}\right), \Omega_{2}=$ $\operatorname{diag}\left(b_{1}, \ldots, b_{n}\right), a_{i}>0(i=1, \ldots, m)$, and $b_{j}>0(j=$ $1, \ldots, n)$, then there exist unique matrices $S$ and $W$ such that

$$
\left\|\Omega_{1} S+\Omega_{2} W-E\right\|^{2}=\min ,
$$

and $S$ and $W$ can be expressed as

$$
S=0, \quad W=\Omega_{2}^{-1} E .
$$

Let $A, C \in \mathbb{C}^{m \times n}, B, D \in \mathbb{C}^{n \times l}$, and $\operatorname{rank} A=p \geq \operatorname{rank} B=$ q. According to Lemma 4, there exist a unitary matrix $U \in$ $\mathbb{C}^{n \times n}$ and nonsingular matrices $R_{A} \in \mathbb{C}^{m \times m}$ and $R_{B} \in \mathbb{C}^{l \times l}$, such that the CCD of matrix pair $\left[A^{*}, B\right]$ is given as

$$
A^{*}=U\left(\Sigma_{A}, 0\right) R_{A}^{-1}, \quad B=U\left(\Sigma_{B}, 0\right) R_{B}^{-1},
$$

where $\Sigma_{A} \in \mathbb{C}^{n \times p}, \Sigma_{B} \in \mathbb{C}^{n \times q}$,

$$
\Sigma_{A}=\left(\begin{array}{ccc}
I_{r} & 0 & 0 \\
0 & G_{s} & 0 \\
0 & 0 & 0 \\
0 & 0 & 0 \\
0 & S_{s} & 0 \\
0 & 0 & I_{t}
\end{array}\right), \quad \Sigma_{B}=\left(\begin{array}{ccc}
I_{r} & 0 & 0 \\
0 & I_{s} & 0 \\
0 & 0 & I_{q-r-s} \\
0 & 0 & 0 \\
0 & 0 & 0 \\
0 & 0 & 0
\end{array}\right)
$$

where $p=r+s+t$,

$$
\begin{aligned}
& G_{s}=\operatorname{diag}\left(g_{r+1}, \ldots, g_{r+s}\right), \quad 1>g_{r+1} \geq \cdots \geq g_{r+s}>0, \\
& S_{s}=\operatorname{diag}\left(w_{r+1}, \ldots, w_{r+s}\right), \quad 0>w_{r+1} \geq \cdots \geq w_{r+s}>1 \text {, } \\
& G_{s}^{2}+S_{s}^{2}=I_{s} \\
& U=\left(\begin{array}{ccccrc}
u_{1} & u_{2} & u_{3} & u_{4} & u_{5} & u_{6}
\end{array}\right) .
\end{aligned}
$$

Without loss of generality, let $p=q$, and then we have the following results.

Theorem 9. Let $A, C \in \mathbb{C}^{m \times n}, B, D \in \mathbb{C}^{n \times l}$, and the CCD of matrix pair $\left[A^{*}, B\right]$ be expressed as (28), and then one has the following.

(a) The least-square solutions to the system (1) are 


$$
X=U\left(\begin{array}{cccccc}
C_{11}+D_{11} & C_{12}+D_{12} & C_{13}+D_{13} & C_{14} & C_{15} & C_{16} \\
D_{21} & D_{22} & D_{23} & 0 & 0 & 0 \\
D_{31} & D_{32} & D_{33} & X_{34} & X_{35} & X_{36} \\
D_{41} & D_{42} & D_{43} & X_{44} & X_{45} & X_{46} \\
Y_{51} & Y_{52} & Y_{53} & S^{-1} C_{24} & S^{-1} C_{25} & S^{-1} C_{26} \\
D_{31}+D_{61} & D_{32}+D_{62} & D_{33}+D_{63} & C_{34} & C_{35} & C_{36}
\end{array}\right) U^{*}
$$

where $X_{34}, X_{35}, X_{36}, X_{44}, X_{45}$, and $X_{46}$ are arbitrary, $Y_{5 i}=$ $\Phi *\left(S\left(G D_{2 i}-C_{2 i}\right)+D_{5 i}\right), i=1,2,3, \Phi=\left(1 /\left(w_{r+j}^{2}+e_{k}^{2}\right)\right) \epsilon$ $\mathbb{C}^{s \times s}$, and $e_{k}=1, j=1, \ldots, s, k=1, \ldots, s$. (b) For arbitrary $X_{34}, X_{35}, X_{36}, X_{44}, X_{45}$, and $X_{46}$, there exists a least-square solution in $\mathbb{C}^{n \times n}$ of (1) which is * congruent to

$$
Y=\left(\begin{array}{cccccc}
C_{11}+D_{11} & C_{12}+D_{12} & C_{13}+D_{13} & C_{14} & C_{15} & C_{16} \\
D_{21} & D_{22} & D_{23} & 0 & 0 & 0 \\
D_{31} & D_{32} & D_{33} & X_{34} & X_{35} & X_{36} \\
D_{41} & D_{42} & D_{43} & X_{44} & X_{45} & X_{46} \\
Y_{51} & Y_{52} & Y_{53} & S^{-1} C_{24} & S^{-1} C_{25} & S^{-1} C_{26} \\
D_{31}+D_{61} & D_{32}+D_{62} & D_{33}+D_{63} & C_{34} & C_{35} & C_{36}
\end{array}\right),
$$

where $Y_{5 i}=\Phi *\left(S\left(G D_{2 i}-C_{2 i}\right)+D_{5 i}\right), i=1,2,3, \Phi=$ $\left(1 /\left(w_{r+j}^{2}+e_{k}^{2}\right)\right) \in \mathbb{C}^{s \times s}$, and $e_{k}=1, j=1, \ldots, s, k=1, \ldots, s$. (c) There exists a minimum norm least-square solution in $\mathbb{C}^{n \times n}$ of (1) which is ${ }^{*}$ congruent to

$$
Y=\left(\begin{array}{cccccc}
C_{11}+D_{11} & C_{12}+D_{12} & C_{13}+D_{13} & C_{14} & C_{15} & C_{16} \\
D_{21} & D_{22} & D_{23} & 0 & 0 & 0 \\
D_{31} & D_{32} & D_{33} & 0 & 0 & 0 \\
D_{41} & D_{42} & D_{43} & 0 & 0 & 0 \\
Y_{51} & Y_{52} & Y_{53} & S^{-1} C_{24} & S^{-1} C_{25} & S^{-1} C_{26} \\
D_{31}+D_{61} & D_{32}+D_{62} & D_{33}+D_{63} & C_{34} & C_{35} & C_{36}
\end{array}\right),
$$

where $Y_{5 i}=\Phi *\left(S\left(G D_{2 i}-C_{2 i}\right)+D_{5 i}\right), i=1,2,3, \Phi=$ $\left(1 /\left(w_{r+j}^{2}+e_{k}^{2}\right)\right) \in \mathbb{C}^{s \times s}$, and $e_{k}=1, j=1, \ldots, s, k=1, \ldots, s$.

Proof. It follows from (28) that

$$
\begin{gathered}
A X=C \Longleftrightarrow\left(R_{A}^{-1}\right)^{*}\left(\begin{array}{c}
\Sigma_{A}^{*} \\
0
\end{array}\right) U^{*} X=C \\
\Longleftrightarrow\left(\begin{array}{c}
\Sigma_{A}^{*} \\
0
\end{array}\right) U^{*} X=\left(R_{A}\right)^{*} C, \\
X B=D \Longleftrightarrow X U\left(\Sigma_{B}, 0\right) R_{B}^{-1}=D \Longleftrightarrow X U\left(\Sigma_{B}, 0\right)=D R_{B} .
\end{gathered}
$$

Then,

$$
\begin{aligned}
\| A X & -C\left\|^{2}+\right\| X B-D \|^{2} \\
= & \left\|\left(\begin{array}{c}
\Sigma_{A}^{*} \\
0
\end{array}\right) U^{*} X-\left(R_{A}\right)^{*} C\right\|^{2}+\left\|X U\left(\Sigma_{B}, 0\right)-D R_{B}\right\|^{2} \\
= & \left\|\left(\begin{array}{c}
\Sigma_{A}^{*} \\
0
\end{array}\right) U^{*} X U-\left(R_{A}\right)^{*} C U\right\|^{2} \\
& +\left\|U^{*} X U\left(\Sigma_{B}, 0\right)-U^{*} D R_{B}\right\|^{2} .
\end{aligned}
$$


Assume that

$$
\begin{gathered}
U^{*} X U=\left(\begin{array}{llllll}
X_{11} & X_{12} & X_{13} & X_{14} & X_{15} & X_{16} \\
X_{21} & X_{22} & X_{23} & X_{24} & X_{25} & X_{26} \\
X_{31} & X_{32} & X_{33} & X_{34} & X_{35} & X_{36} \\
X_{41} & X_{42} & X_{43} & X_{44} & X_{45} & X_{46} \\
X_{51} & X_{52} & X_{53} & X_{54} & X_{55} & X_{56} \\
X_{61} & X_{62} & X_{63} & X_{64} & X_{65} & X_{66}
\end{array}\right), \\
\left(R_{A}\right)^{*} C U=\left(\begin{array}{llllll}
C_{11} & C_{12} & C_{13} & C_{14} & C_{15} & C_{16} \\
C_{21} & C_{22} & C_{23} & C_{24} & C_{25} & C_{26} \\
C_{31} & C_{32} & C_{33} & C_{34} & C_{35} & C_{36} \\
C_{41} & C_{42} & C_{43} & C_{44} & C_{45} & C_{46}
\end{array}\right), \\
U^{*} D R_{B}=\left(\begin{array}{lllll}
D_{11} & D_{12} & D_{13} & D_{14} \\
D_{21} & D_{22} & D_{23} & D_{24} \\
D_{31} & D_{32} & D_{33} & D_{34} \\
D_{41} & D_{42} & D_{43} & D_{44} \\
D_{51} & D_{52} & D_{53} & D_{54} \\
D_{61} & D_{62} & D_{63} & D_{64}
\end{array}\right),
\end{gathered}
$$

and then

$$
\begin{aligned}
& \left\|\left(\begin{array}{c}
\Sigma_{A}^{*} \\
0
\end{array}\right) U^{*} X U-\left(R_{A}\right)^{*} C U\right\|^{2}+\left\|U^{*} X U\left(\Sigma_{B}, 0\right)-U^{*} D R_{B}\right\|^{2} \\
& =\|\left(\begin{array}{cccc}
X_{11} & X_{12} & \cdots & X_{16} \\
G X_{21}+S X_{51} & G X_{22}+S X_{52} & \cdots & G X_{26}+S X_{56} \\
X_{61} & X_{62} & \cdots & X_{66} \\
0 & 0 & \cdots & 0
\end{array}\right) \\
& -\left(\begin{array}{llll}
C_{11} & C_{12} & \cdots & C_{16} \\
C_{21} & C_{22} & \cdots & C_{26} \\
C_{31} & C_{32} & \cdots & C_{36} \\
C_{41} & C_{42} & \cdots & C_{46}
\end{array}\right) \|^{2} \\
& +\left(\begin{array}{cccc}
X_{11} & X_{12} & X_{13} & 0 \\
X_{21} & X_{22} & X_{23} & 0 \\
\vdots & \vdots & \vdots & \vdots \\
X_{61} & X_{62} & X_{63} & 0
\end{array}\right) \\
& -\left(\begin{array}{cccc}
D_{11} & D_{12} & D_{13} & D_{14} \\
D_{21} & D_{22} & D_{23} & D_{24} \\
\vdots & \vdots & \vdots & \vdots \\
D_{61} & D_{62} & D_{63} & D_{64}
\end{array}\right) \|^{2} \\
& =\left\|X_{11}-C_{11}\right\|^{2}+\left\|X_{11}-D_{11}\right\|^{2}+\left\|X_{12}-C_{12}\right\|^{2} \\
& +\left\|X_{12}-D_{12}\right\|^{2}+\left\|X_{13}-C_{13}\right\|^{2}+\left\|X_{13}-D_{13}\right\|^{2} \\
& +\left\|X_{61}-C_{31}\right\|^{2}+\left\|X_{61}-D_{61}\right\|^{2}+\left\|X_{62}-C_{32}\right\|^{2} \\
& +\left\|X_{62}-D_{62}\right\|^{2}+\left\|X_{63}-C_{33}\right\|^{2}+\left\|X_{63}-D_{63}\right\|^{2}
\end{aligned}
$$

$$
\begin{aligned}
& +\left\|G X_{21}+S X_{51}-C_{21}\right\|^{2}+\left\|X_{21}-D_{21}\right\|^{2} \\
& +\left\|X_{51}-D_{51}\right\|^{2}+\left\|G X_{22}+S X_{52}-C_{22}\right\|^{2} \\
& +\left\|X_{22}-D_{22}\right\|^{2}+\left\|X_{52}-D_{52}\right\|^{2} \\
& +\left\|G X_{23}+S X_{53}-C_{23}\right\|^{2}+\left\|X_{23}-D_{23}\right\|^{2} \\
& +\left\|X_{53}-D_{53}\right\|^{2}+\left\|G X_{24}+S X_{54}-C_{24}\right\|^{2} \\
& +\left\|G X_{25}+S X_{55}-C_{25}\right\|^{2}+\left\|G X_{26}+S X_{56}-C_{26}\right\|^{2} \\
& +\left\|X_{14}-C_{14}\right\|^{2}+\left\|X_{15}-C_{15}\right\|^{2}+\left\|X_{16}-C_{16}\right\|^{2} \\
& +\left\|X_{64}-C_{34}\right\|^{2}+\left\|X_{65}-C_{35}\right\|^{2}+\left\|X_{66}-C_{36}\right\|^{2} \\
& +\left\|X_{31}-D_{31}\right\|^{2}+\left\|X_{32}-D_{32}\right\|^{2}+\left\|X_{33}-D_{33}\right\|^{2} \\
& +\left\|X_{41}-D_{41}\right\|^{2}+\left\|X_{42}-D_{42}\right\|^{2}+\left\|X_{43}-D_{43}\right\|^{2} .
\end{aligned}
$$

By Lemmas 5, 7, and 8, a general form of the least-square solutions can be expressed as (31); for arbitrary $X_{34}, X_{35}, X_{36}$, $X_{44}, X_{45}$, and $X_{46}$, there exists a least-square solution in $\mathbb{C}^{n \times n}$ of (1) which is * congruent to (32), and the part (c) follows from the definition of Frobenius norm.

\section{An Algorithm and Numerical Examples}

Based on the main results of this paper, we in this section propose an algorithm for finding the least-square solutions to the system (1). All the tests are performed by MATLAB 6.5 which has a machine precision of around $10^{-16}$.

Algorithm 1. (1) Input $A \in \mathbb{C}^{m \times n}$ and $B \in \mathbb{C}^{n \times l}$, and compute $U \in \mathbb{C}^{n \times n}, R_{A}^{-1} \in \mathbb{C}^{m \times m}, R_{B}^{-1} \in \mathbb{C}^{l \times l}, \Sigma_{A}, \Sigma_{B} \in$ $\mathbb{C}^{n \times p}$, and $G, S \in \mathbb{C}^{s \times s}$ by the CCD of matrix pair $\left[A^{*}, B\right]$.

(2) Input $C \in \mathbb{C}^{m \times n}, D \in \mathbb{C}^{n \times l}$, and compute $C_{i j}(i=$ $1,2,3,4 ; j=1,2,3,4,5,6)$ and $D_{l k}(l=1,2,3,4,5,6 ; k=$ $1,2,3,4)$ according to (37).

(3) Compute the least-square solutions of the system (1) by (31).

(4) Compute the ${ }^{*}$ congruence class of the least-square and the minimum norm least-square solutions to the system (1) according to (32) and (33).

Example 1. Suppose

$$
\begin{aligned}
A=\left[\begin{array}{cccccc}
-1.625 & 0 & -0.6875 i & 0.875 i & 0.3438 & 0 \\
-2 & 0 & -0.5 i & 0.875 i & 0.25 & 0 \\
-0.75 & 0 & -0.125 i & 0.25 i & 0.0625 & 0 \\
2.625 & 0 & 0.6875 i & -0.875 i & -0.3438 & 0
\end{array}\right], \\
B= \\
=\left[\begin{array}{cccc}
2 & -2 & 0 & -1 \\
-5 i & 6 i & 0 & 2 i \\
31 i & -37 i & i & -15 i \\
0 & 0 & 0 & 0 \\
0 & 0 & 0 & 0 \\
0 & 0 & 0 & 0
\end{array}\right]
\end{aligned}
$$




$$
\begin{gathered}
C=\left[\begin{array}{cccccc}
3 & 2 & 2 & 1 & 1 & 5 \\
4 & i & i & -i & 3 & 1 \\
5 & 6 & 7 & 4 & 3 & 1 \\
2 & 1 & 1 & 4 & 3 & 4
\end{array}\right], \\
D=\left[\begin{array}{cccc}
i & -i & -i & 1 \\
2 & 3 & 1 & 4 \\
4 & 1 & 2 & 3 \\
5 & 7 & 6 & 9 \\
9 & 7 & 5 & 6 \\
1 & 2 & 3 & 2
\end{array}\right] .
\end{gathered}
$$

Applying Algorithm 1, we obtain the following:

$$
\begin{gathered}
U=\left[\begin{array}{cccccc}
1 & 0 & 0 & 0 & 0 & 0 \\
0 & 0 & -i & 0 & 0 & 0 \\
0 & i & 0 & 0 & 0 & 0 \\
0 & 0 & 0 & 0 & 0 & i \\
0 & 0 & 0 & 0 & 1 & 0 \\
0 & 0 & 0 & -i & 0 & 0
\end{array}\right], \\
\Sigma_{A}=\left[\begin{array}{ccc}
1 & 0 & 0 \\
0 & 0.5 & 0 \\
0 & 0 & 0 \\
0 & 0 & 0 \\
0 & 0.25 & 0 \\
0 & 0 & 1
\end{array}\right], \\
\Sigma_{B}=\left[\begin{array}{lll}
1 & 0 & 0 \\
0 & 1 & 0 \\
0 & 0 & 1 \\
0 & 0 & 0 \\
0 & 0 & 0 \\
0 & 0 & 0
\end{array}\right],
\end{gathered}
$$

$$
\begin{gathered}
R_{A}^{-1}=\left[\begin{array}{cccc}
-1.625 & -2 & -0.75 & 2.625 \\
1.375 & 1 & 0.25 & -1.375 \\
-0.875 & 0 & -0.25 & 0.875 \\
0.375 & 0 & 0.25 & -0.375
\end{array}\right], \\
R_{B}^{-1}=\left[\begin{array}{cccc}
2 & -2 & 0 & -1 \\
31 & -37 & 1 & -15 \\
5 & -6 & 0 & -2 \\
-44 & 52 & -1 & 21
\end{array}\right] \\
G=[0.5],
\end{gathered}
$$

$$
\begin{gathered}
\left(R_{A}\right)^{*} C U=\left(\begin{array}{cccccc}
5 & 3 i & -3 i & -9 i & 4 & 5 i \\
14 & -1+6 i & 1-6 i & -19 i & 11 & 1+10 i \\
32 & -2+26 i & 2-23 i & -22 i & 25 & 2+25 i \\
53 & -1+57 i & 1-50 i & -34 i & 38 & 1+46 i
\end{array}\right), \\
U^{*} D R_{B}=\left(\begin{array}{ccccc}
0.03-0.05 i & 0.02-0.05 i & 0.04-0.02 i & 0.02-0.04 i \\
-0.51 i & -0.27 i & -0.33 i & -0.25 i \\
0.43 i & 0.21 i & 0.29 i & 0.2 i \\
0.41 i & 0.26 i & 0.25 i & 0.23 i \\
1.35 & 0.67 & 0.78 & 0.62 \\
-1.27 i & -0.71 i & -0.82 i & -0.65 i
\end{array}\right) .
\end{gathered}
$$

The least-square solutions to the system (1) are

$$
X=U\left(\begin{array}{cccccc}
5.03-0.05 i & 0.02+2.95 i & 0.04-3.02 i & -9 i & 4 & 5 i \\
-0.51 i & -0.27 i & -0.33 i & 0 & 0 & 0 \\
0.43 i & 0.21 i & 0.29 i & X_{34} & X_{35} & X_{36} \\
0.41 i & 0.26 i & 0.25 i & X_{44} & X_{45} & X_{46} \\
2.02-0.06 i & 0.864-0.366 i & 0.498+1.448 i & 76 i & 44 & 4+40 i \\
-0.84 i & -0.5 i & -0.53 i & -22 i & 25 & 2+25 i
\end{array}\right) U^{*}
$$

where $X_{34}, X_{35}, X_{36}, X_{44}, X_{45}$, and $X_{46}$ are arbitrary.

For arbitrary $X_{34}, X_{35}, X_{36}, X_{44}, X_{45}$, and $X_{46}$, there exists a least-square solution in $\mathbb{C}^{6 \times 6}$ of (1) which is ${ }^{*}$ congruent to

$$
Y=\left(\begin{array}{cccccc}
5.03-0.05 i & 0.02+2.95 i & 0.04-3.02 i & -9 i & 4 & 5 i \\
-0.51 i & -0.27 i & -0.33 i & 0 & 0 & 0 \\
0.43 i & 0.21 i & 0.29 i & X_{34} & X_{35} & X_{36} \\
0.41 i & 0.26 i & 0.25 i & X_{44} & X_{45} & X_{46} \\
2.02-0.06 i & 0.864-0.366 i & 0.498+1.448 i & 76 i & 44 & 4+40 i \\
-0.84 i & -0.5 i & -0.53 i & -22 i & 25 & 2+25 i
\end{array}\right) .
$$

There exists a minimum norm least-square solution in $\mathbb{C}^{6 \times 6}$ of (1) which is * congruent to

$$
Y=\left(\begin{array}{cccccc}
5.03-0.05 i & 0.02+2.95 i & 0.04-3.02 i & -9 i & 4 & 5 i \\
-0.51 i & -0.27 i & -0.33 i & 0 & 0 & 0 \\
0.43 i & 0.21 i & 0.29 i & 0 & 0 & 0 \\
0.41 i & 0.26 i & 0.25 i & 0 & 0 & 0 \\
2.02-0.06 i & 0.864-0.366 i & 0.498+1.448 i & 76 i & 44 & 4+40 i \\
-0.84 i & -0.5 i & -0.53 i & -22 i & 25 & 2+25 i
\end{array}\right)
$$




\section{Conflict of Interests}

The authors declare that there is no conflict of interests regarding the publication of this paper.

\section{Acknowledgments}

This research was supported by the Youth Funds of Natural Science Foundation of Hebei province (A2012403013), the Education Department Foundation of Hebei province (Z2013110), and the Natural Science Foundation of Hebei province (A2012205028).

\section{References}

[1] R. A. Horn, V. V. Sergeichuk, and N. Shaked-Monderer, "Solution of linear matrix equations in a congruence class," The Electronic Journal of Linear Algebra, vol. 13, pp. 153-156, 2005.

[2] C. G. Khatri and S. K. Mitra, "Hermitian and nonnegative definite solutions of linear matrix equations," SIAM Journal on Applied Mathematics, vol. 31, no. 4, pp. 579-585, 1976.

[3] F. J. H. Don, "On the symmetric solutions of a linear matrix equation," Linear Algebra and its Applications, vol. 93, pp. 1-7, 1987.

[4] H. Dai, "On the symmetric solutions of linear matrix equations," Linear Algebra and its Applications, vol. 131, pp. 1-7, 1990.

[5] A. Dajić and J. J. Koliha, "Equations $a x=c$ and $x b=d$ in rings and rings with involution with applications to Hilbert space operators," Linear Algebra and Its Applications, vol. 429, no. 7, pp. 1779-1809, 2008.

[6] H. X. Chang and Q. W. Wang, "Reflexive solution to a system of matrix equations," Journal of Shanghai University, vol. 11, no. 4, pp. 355-358, 2007.

[7] Q. W. Wang and C. Z. Dong, "The positive solution to a system of adjointable operator equations over Hilbert $C^{*}$-modules," Linear Algebra and Its Applications, vol. 433, pp. 1481-1489, 2010.

[8] C.-Z. Dong, Q.-W. Wang, and Y.-P. Zhang, “The common positive solution to adjointable operator equations with an application," Journal of Mathematical Analysis and Applications, vol. 396, no. 2, pp. 670-679, 2012.

[9] C. Z. Dong, Q. W. Wang, and Y. P. Zhang, "On the hermitian $R$-conjugate solution of a system of Matrix equations," Journal of Applied Mathematics, Article ID 398085, 14 pages, 2012.

[10] H. X. Chang, Q. W. Wang, and G. J. Song, " $(R, S)$-conjugate solution to a pair of linear matrix equations," Applied Mathematics and Computation, vol. 217, no. 1, pp. 73-82, 2010.

[11] Z. Y. Peng, Y. B. Deng, and J. W. Liu, "Least-squares solution of inverse problem for Hermitian anti-reflexive matrices and its approximation," Acta Mathematica Sinica, vol. 22, no. 2, pp. 477-484, 2006.

[12] Y. H. Liu, "Ranks of least squares solutions of the matrix equation $A X B=C$," Computers \& Mathematics with Applications, vol. 55, no. 6, pp. 1270-1278, 2008.

[13] S. Yuan and Q. Wang, "Two special kinds of least squares solutions for the quaternion matrix equation $A X B+C X D=E$," Electronic Journal of Linear Algebra, vol. 23, pp. 257-274, 2012.

[14] S.-F. Yuan, Q.-W. Wang, and X. Zhang, "Least-squares problem for the quaternion matrix equation $A X B+C Y D=E$ over different constrained matrices," International Journal of Computer Mathematics, vol. 90, no. 3, pp. 565-576, 2013.
[15] Q. W. Wang and J. Yu, "On the generalized bi (skew-) symmetric solutions of a linear matrix equation and its procrust problems," Applied Mathematics and Computation, vol. 219, no. 19, pp. 9872-9884, 2013.

[16] I. Kyrchei, "Analogs of Cramer's rule for the minimum norm least squares solutions of some matrix equations," Applied Mathematics and Computation, vol. 218, no. 11, pp. 6375-6384, 2012.

[17] B. Zheng, L. Ye, and D. S. Cvetkovic-Ilic, "The * congruence class of the solutions of some matrix equations," Computers \& Mathematics with Applications, vol. 57, no. 4, pp. 540-549, 2009.

[18] G. Xu, M. Wei, and D. Zheng, "On solutions of matrix equation $A X B+C Y D=F$, Linear Algebra and Its Applications, vol. 279, no. 1-3, pp. 93-109, 1998. 


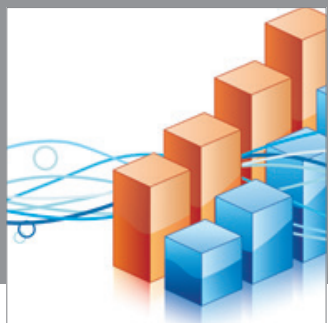

Advances in

Operations Research

mansans

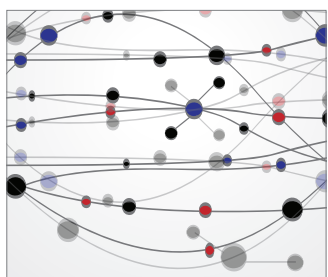

The Scientific World Journal
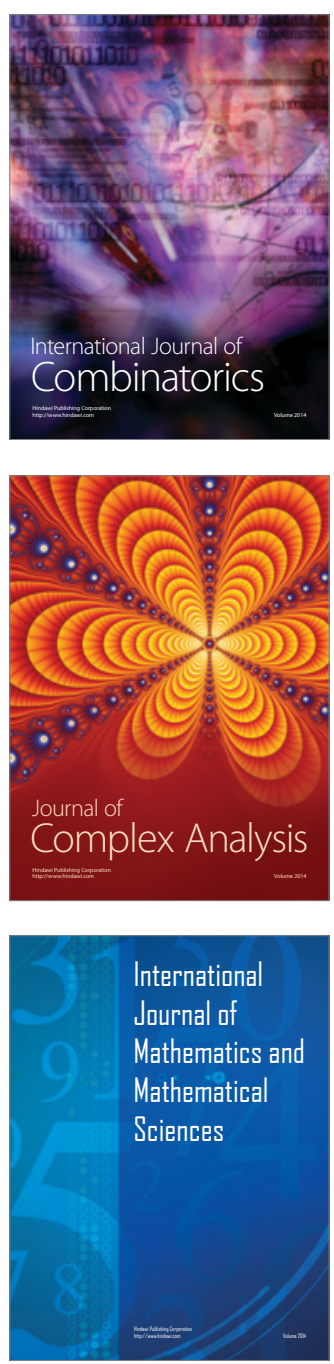
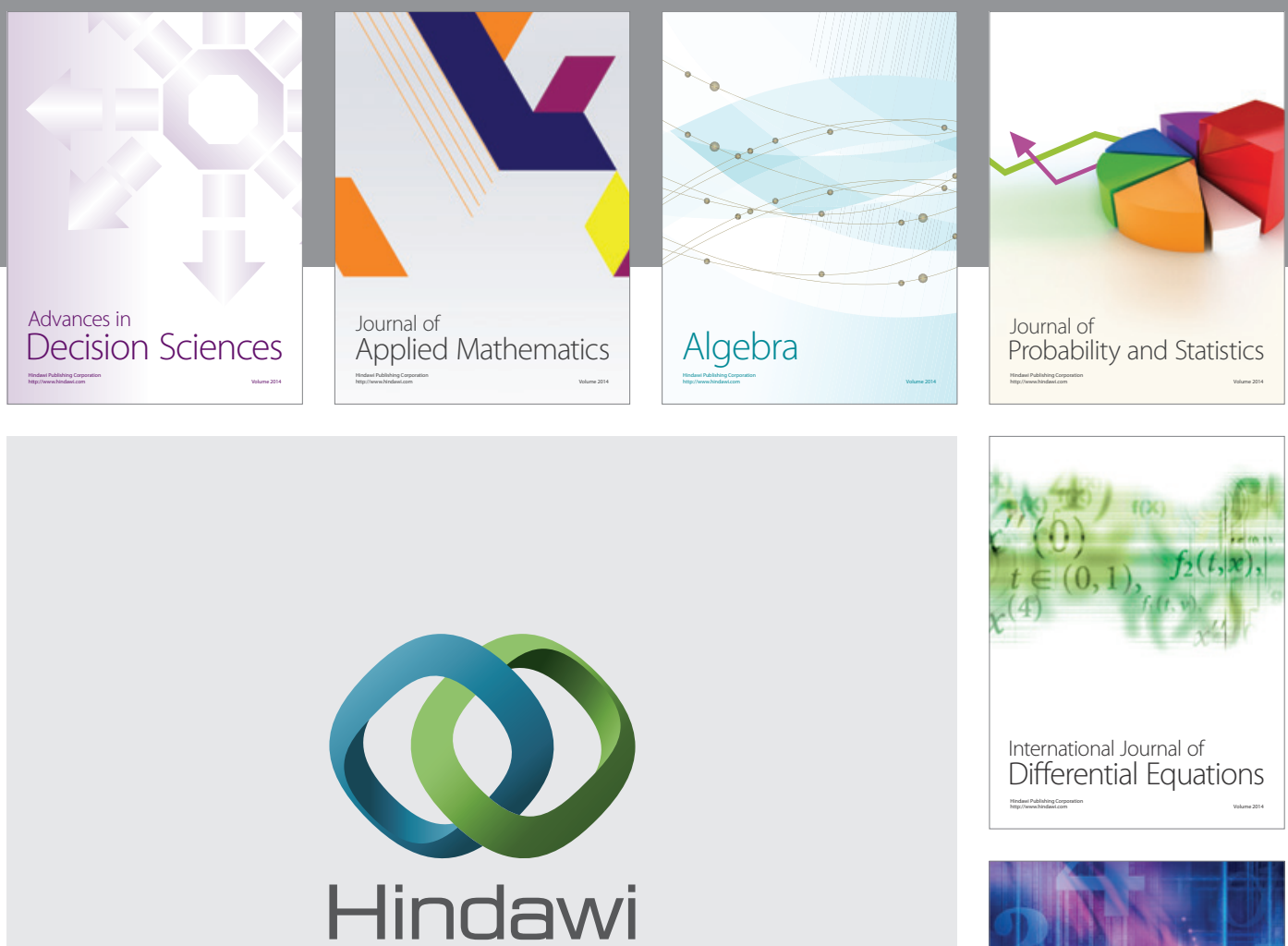

Submit your manuscripts at http://www.hindawi.com
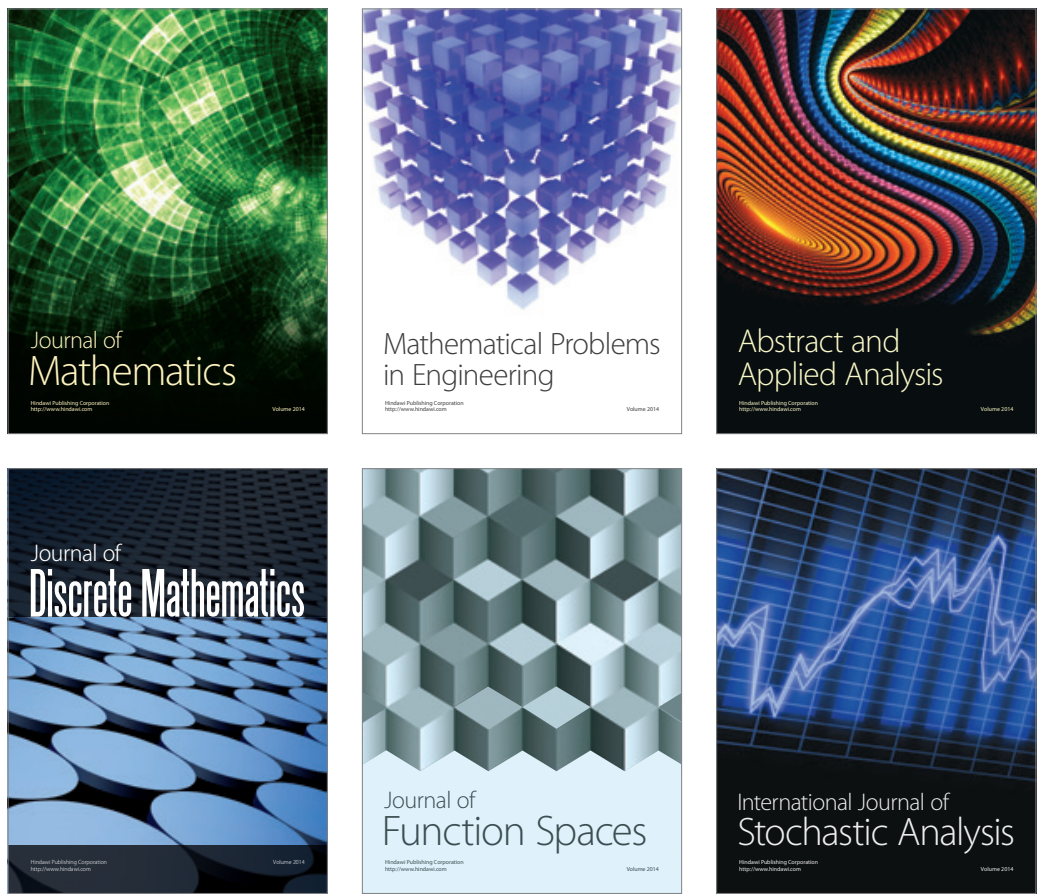

Journal of

Function Spaces

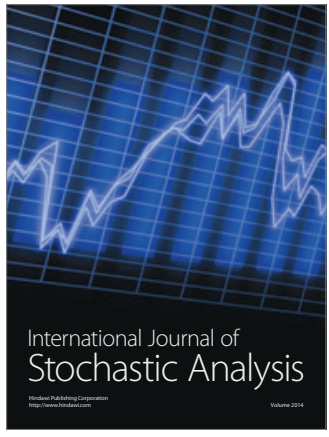

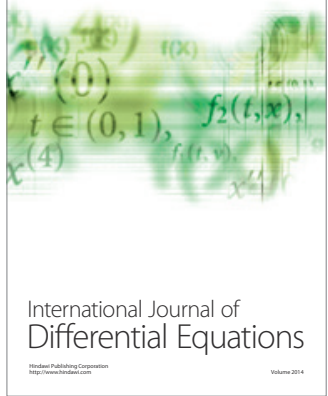
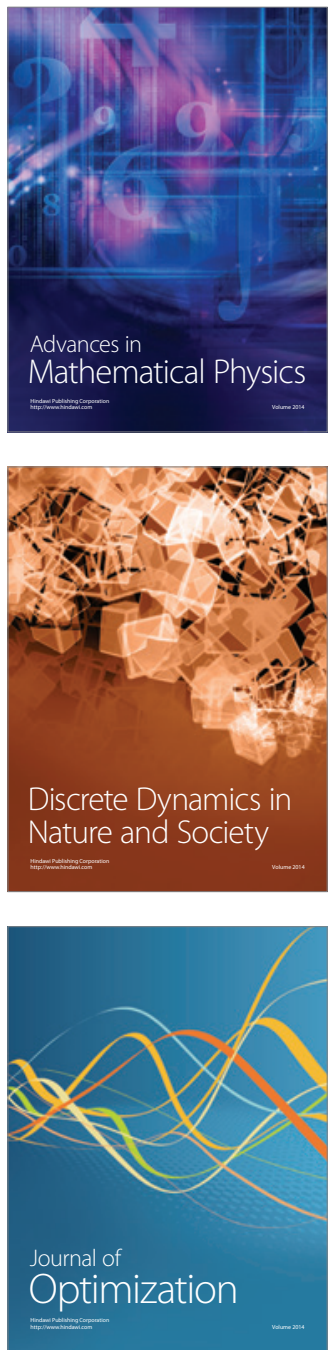